\title{
Design and Application of a New Artificial Intelligence Screening Program for Scoliosis
}

\author{
Yichen Li \\ The Experimental High School Attached to Beijing Normal University, Beijing 100032, China \\ DOI: $10.32629 / \mathrm{jcmr} . v 2 \mathrm{i} 4.548$
}

\begin{abstract}
Objective - Intelligent Scoliosis screening Application (ISSAPP) is an intelligent scoliosis screening application based on back photos, which is used to determine the effectiveness and reliability of the new tool and reduce the cost of screening by comparing it with traditional physical examination. Method — To design and upgrade the measurement procedures for back photographs during physical examination, including the measurement of bilateral shoulder apex line and horizon plus angle, and the measurement of the angle between the thoracic apex line and horizon during Adams' spinal flexion experiment so as to determine the validity and reliability of measurement program tools. Results - The first and second versions of the designed AI measurement program were more sensitive than the traditional physical examination, Adams spinal flexion test, and lateral flexion instrument. The majority of patients who were tested positive using the measurement procedure tool met the requirements for diagnosis of scoliosis. Conclusions - This new AI based measurement and screening tool can be used for early detection of shoulder and thoracic malformation caused by scoliosis, which is a simple, convenient and sensitive early screening tool. Based on this development idea, an upgraded version can be further developed for early screening of scoliosis.
\end{abstract}

Keywords: scoliosis, screening application, image recognition

\section{Introduction}

Scoliosis is a complex three-dimensional deformity of the spine caused by a variety of etiologies, which leads to the development of the bony structure and organs around the spine, followed by a variety of physical and psychological diseases. Idiopathic Scoliosis, also known as Adolescent Idiopathic Scoliosis, is the most common form, with a prevalence rate of $0.47 \%$ to $5.2 \%$ in adolescents around the world ${ }^{[1-2]}$. It varies by age and gender, which is most common in girls. The incidence of scoliosis in girls aged 10-12 years showed the highest occurrence of 5.57\%, and the domestic incidence is higher at $0.11-12.05 \%{ }^{[3-4]}$. The pathogenesis is unknown and may be related to genetics, hormones and endocrinology.

More efforts have been made in scoliosis screening at home and abroad. The Governments of European and American countries and China have carried out screening work successively, and designated clinical practice guidelines and government working standards. Commonly adopted screening methods usually include physical examination, Adams flexion test, spinal ruler, moire photography ${ }^{[5-7]}$, etc. Recently, deep learning screening technology based on the appearance of human bare back has been reported ${ }^{[8]}$. There are some differences in the sensitivity, specificity and convenience of different methods reported at home and abroad.

Based on the progress of information age, artificial intelligence and mobile phone operating system, engineers and doctors made a joint effort to design a mobile phone program based on back photo measurement to measure thoracic deformity caused by spinal curvature, effectively protecting the privacy of parents and children. The effectiveness and reliability of the new tool can be determined by comparing patients in schools and hospitals, which can reduce the cost of screening on the one hand and provide new screening ideas on the other hand.

\section{Data and methods}

\subsection{ISSAPP based principle and operation process}

The improvement of the accuracy and simplification of operation of mobile phone photography brings convenience and privacy protection to a lot of scientific researches, which can be operated by parents of teenagers at home. As the presence of thoracic rotation and developmental malformation caused by scoliosis, problems such as unequal height of shoulders and thoracic asymmetry are prone to occur. In the physical examination process, the back image is imported into the mobile phone program, and the analysis software is applied to determine valgus and rotation angle, so as to define the thoracic malformation, and then infer the presence of scoliosis in the patient. 
Basic requirements of ISSAPP 1.0: Testees will be photographed with their back to the phone in a standard position of underwear (boys or bare back) facing a single background color. To make sure that the line between the testees' shoulders is more than 5 degrees from the horizon as positive. Four Adams experiments can be added to ISSAPP2.0 on the basis of 1.0 version. Consistent requirements on photography are made with testees required to perform Adams experiments of different level. The subjects placed their hands at the middle part of the thigh, patella, middle part of the calf and ankle joint, and conducted Adams experiments in four different parts. Four photos were obtained. The connection between the highest points of the bilateral thorax was determined, and the included angle between the connection and the horizon was measured.

\subsection{ISSAPP reliability and validity test}

From 2020 to 2021, spinal surgeons were invited to screen 750 students aged 13-17 from two high schools and one junior high school in Xicheng District, Beijing, using physical examination, Adams test and scoliosis measuring scales and ISSAPP.

\section{Results}

ISSAPP based design requires mobile phones to have high pixel requirements, not less than 8 million pixels with image quality not be less than $2 \mathrm{M}$. Besides, a single light color is a must in photography background and Subjects need to wear tight dark underwear or boys need to be naked back, mobile phone running program can be downloaded in advance for operation.

Table 1. ISSAPP and screening results by physical examination and measurement

\begin{tabular}{ccccccc}
\hline \multirow{2}{*}{ Indicators } & \multicolumn{3}{c}{ ISSAPP } & \multicolumn{2}{c}{ Physical inspection and measuring ruler } \\
\cline { 2 - 6 } & Male & Female & Total & Male & Female \\
\hline Testee & 370 & 380 & 750 & 370 & 380 & 750 \\
Suspect & 11 & 20 & 31 & 10 & 26 & 36 \\
Suspicious rate & 2.9 & 5.2 & 4.1 & 2.7 & 6.8 & 4.8 \\
\hline
\end{tabular}

According to a report in 1997[9], 5 degree is used as a reference value for positive spinal ruler. ISSAPP found 31 suspicious objects, and 36 suspicious objects were detected by physical screening and measuring ruler, with similar detection results.

\section{Discussion}

Based on advances in science and technology, application and development of mobile phone and preliminary screening, we developed a new artificial intelligence curve screening software, which has been preliminarily verified in school screening. It is indeed non-invasive, effective, reliable and sensitive, and can well protect the privacy of the tested. ISSAPP is simple and easy to learn. After simple learning procedures, parents can complete preliminary screening at home. For people in remote areas, it can greatly save social resources and reduce testing costs.

Definitely, the research and development of this method is based on the unequal height of shoulder and thoracic deformity caused by scoliosis. The human body has a certain ability to correct, and the corresponding spine will have a certain compensatory curvature, which may have a certain degree of missed diagnosis. Thoracic malformation is also a compensatory manifestation of patients' disease progression to a certain extent. Most of the patients tested positive are patients with severe disease progression to a certain extent. Therefore, ISSAPP needs to be further upgraded to increase the detection of different parameters such as light intensity and morphology to increase the sensitivity of screening. Additional data can be obtained by continuing to expand the number of students screened, and the accuracy and specificity of ISSAPP can be determined by correlation studies of radio-confirmed cases.

In addition, in light of ISSAPP, photos can be obtained by mobile phone, as a result, there should be certain balance and shooting requirements for the operator when taking photos. The positive standard of 5 degree can also reduce the error of mobile phone shooting. How mobile measurement based software can better determine the line and angle and increase accuracy is also the direction of future research and development.

ISSAPP is an attempt to improve the physical detection method of lateral bending under the new situation. Through practical verification, it proves good accuracy, sensitivity and specificity, which is simple for operation, and can be used for protection of the privacy of the subject, so it can be further developed and studied. 


\section{References}

[1] Weinstein S L, Dolan L A, Cheng J C, et al. Adolescent idiopathic scoliosis[J]. Lancet, 2008, 371(9623): $1527-1537$.

[2] Aulisa A G, Giordano M, Guzzanti V, et al. Effectiveness of school scoliosis screening and the importance of this method in measures to reduce morbidity in an Italian territory[J]. J Pediatr Orthop B, 2019, 28(3): 271-277.

[3] Lu jianmin, Qiu lin, Miller. Moire topography screening for scoliosis. Acta Acad Med Primae Shanghai, 1985, 12: 302304.

[4] Liu SI, Huang DS. Scoliosis in China, A general review. Clin Orthop Relat Res, 1996, 323: 113-118.

[5] Farhaan A, Alexander G, Zaher D, et al. Adolescent idiopathic scoliosis. BMJ, 2013, 346: f2508

[6] Hresko M Timothy. Clinical practice. Idiopathic scoliosis in adolescents. N Engl J Med, 2013, 368: 834-841.

[7] Hiroshi K, Takuya N, Etsuo C, et al. School scoliosis screening by moire tomography- overview for 33 years in Miyazaki Japan. J Orthop Sci, 2018, 23: 609-613.

[8] Yang JL, Zhang K, Fan HW, et al. Development and validation of deep learning algorithms for scoliosis screening using back images. Commun Biol, 2019 Oct 25;2: 390.

[9] Huang SC. Cut-off point of the scoliometer in school scoliosis screening. Spine, 1997; 22: 1985 